\title{
Germanica
}

\section{Im Angesicht des Untergangs : Krisen des Individuums im deutschsprachigen (Post)Desaster- Roman}

Face à la perspective de la fin: Crises de l'individu dans le roman d'après la catastrophe de langue allemande

Facing Doom: Crises of the individual in German (post-) Disaster Novels

\section{Solange Landau}

\section{(2) OpenEdition}

Journals

Édition électronique

URL : http://journals.openedition.org/germanica/2667

DOI : $10.4000 /$ germanica.2667

ISSN : 2107-0784

Éditeur

Université de Lille

Édition imprimée

Date de publication : 30 décembre 2014

Pagination : $97-110$

ISBN : 9782913857346

ISSN : 0984-2632

Référence électronique

Solange Landau, «Im Angesicht des Untergangs : Krisen des Individuums im deutschsprachigen (Post)Desaster-Roman », Germanica [Online], 55 | 2014, Online erschienen am: 30 Dezember 2016, abgerufen am 06 Oktober 2020. URL : http://journals.openedition.org/germanica/2667 ; DOI : https:// doi.org/10.4000/germanica.2667 


\title{
Im Angesicht des Untergangs : Krisen des Individuums im deutschsprachigen (Post)Desaster-Roman
}

\author{
Solange LANDAU \\ Universität des Saarlandes - Saarbrücken
}

Susan Sonntag beschreibt in ihrem Aufsatz „Die Katastrophenphantasie"1 die Faszination des Untergangs: „So geht es [...] um die Ästhetik der Destruktion, um die seltsame Schönheit der rächenden Verwüstung, der Schaffung eines Chaos" 2 . Der Leser oder Zuschauer wird zum Prototyp des Voyeurs, der lediglich betrachtet und sich weniger emotional mit den Helden identifiziert. Schon 1965 kommt sie zu dem Schluss, dass neben der ,Angst vor der physischen Katastrophe, vor der Gefahr einer allgemeinen Verstümmelung und sogar Ausrottung "3, sich in Katastrophenphantasien ,eine starke Beunruhigung über den Zustand der individuellen Psyche“" widerspiegelt.

1. - Susan Sonntag, ,Die Katastrophenphantasie“, in: Dies., Kunst und Antikunst. 24 literarische Analysen. Frankfurt am Main, Fischer Taschenbuch Verlag, 2009, S. 279 298.

2. - Susan Sonntag, a.a.O., S. 283.

3. - Ebd., S. 292.

4. - Ebd.., S. 292. 
Susan Sonntag schrieb diesen Aufsatz zu einer Zeit, als der Kalte Krieg seinen Höhepunkt erreichte und die ständige Angst vor einem nuklearen Desaster fast zum Alltag gehörte. Eine solche Existenzangst, die unterschwellig die Menschen erfasst und medial vorangetrieben wird, findet sich allerdings bis in die heutige Zeit. Nach einer unbestimmten Angst vor dem Milleniumswechsel, die vor allem die Internetvernetzung weltweiter Institutionen wie beispielsweise Banken betraf, erlebte die (post)moderne Gesellschaft ihre bislang tiefste Erschütterung durch die Anschläge vom 11. September 2001 auf die Vereinigten Staaten von Amerika.

Tatsächlich scheint die Menschheit, oder besser gesagt: die gegenwärtige Gesellschaft, wie wir sie kennen, mehr denn je am Rande ihres eigenen Untergangs zu stehen. Zur zunehmenden Angst vor terroristischen Anschlägen, die überall und jederzeit stattfinden können, gesellt sich die Furcht vor unkalkulierbaren Schäden durch Klimaund Naturkatastrophen. Allein in Deutschland verursachen Fluten und Orkane wie beispielsweise Lothar um die Jahrtausendwende und Kyrill im Jahre 2007 Millionenschäden. Man fühlt sich zwar trotz deutscher Militärbeteiligungen im Ausland sicher vor einem Krieg vor der eigenen Haustür, kann sich aber nicht vor unkontrollierbaren Naturereignissen oder terroristischen Attentaten schützen. Somit steigt zwar auf der einen Seite das Katastrophenbewusstsein und die entsprechende Angst mehr und mehr an, zugleich findet aber durch die ständige mediale Präsens eine Banalisierung und auch eine Abstumpfung des Einzelnen statt die alltäglich stattfindenden Desaster werden zunehmend ausgeblendet, während die Faszination an fiktionalen Weltuntergängen, Unglücken und (Post)Apokalypsen ungebrochen scheint. Gleichzeitig werden in der Popkultur zunehmend Ängste geschürt und ausgeschlachtet, etwa vor dem Ende des ominösen Maya-Kalenders im Dezember 2012.

An diese Faszination knüpft Kathrin Röggla mit ihren im Essayband besser wäre: keine ${ }^{5}$ erschienenen Aufsätzen zum Themenkomplex der Katastrophenphantasien an und nimmt dabei zugleich dezidiert Bezug auf Susan Sonntag. Sie definiert die menschlichen Hintergründe jener Lust am Untergang als „Sehnsucht nach einer kathartischen Erfahrung oder aus einem aggressiven Verlangen heraus, im Ausnahmezustand, die bestehende Ordnung gleichzeitig negiert und auf die Spitze getrieben zu sehen" 6 . Doch zugleich stellt sie fest, dass diese Phantasie "keinen externen Ort mehr hat, sondern schon immer um uns ist" ${ }^{\text {"7 }}$. Das Desaster scheint dementsprechend Teil der menschlichen Natur zu sein

5. - Kathrin Röggla, Besser wäre : keine, Frankfurt am Main, Fischer Verlag, 2014.

6. - Kathrin Röggla, „Geisterstädte, Geisterfilme“, in: Dies., a.a.O., S. 7.

7. - Kathrin Röggla, „Die Rückkehr der Körperfresser“, in : Dies., a.a.O., S. 30. 
und dennoch ,,ist [es] der Mensch, der die Katastrophe zur Katastrophe macht" .

Folgt man dieser Definition, ist die Katastrophe also ein menschliches Konstrukt - denn die Natur kennt keine Katastrophen - und zugleich ein Grundbedürfnis, das zu jeder Zeit und überall stattfindet, wobei gerade die Angst davor tief in das menschliche Wesen und das kulturelle Gedächtnis eingebrannt scheint. 9/11, Klimakatastrophen, Wirtschaftskrisen und individuelle Existenzängste vermischen sich und treiben diese eingebrannte Angst vor dem Unbestimmten und vor der eigenen Zukunft auf die Spitze.

Diese wirklichkeitsverändernden Erfahrungen finden somit auch ihren Niederschlag in literarischen Verarbeitungen. Gerade nach den Anschlägen vom 11. September 2001 steht die Menschheit im neuen Millennium vor nie dagewesenen Herausforderungen und Veränderungen in ihrer Zivilisationsstruktur, womit sich auch die jüngere deutschsprachige Schriftstellergeneration in ihren Werken auseinandersetzt. Während Sibylle Bergs Ende gut (2004) ${ }^{9}$ dezidiert die gegenwärtige Gesellschaft mit ihrer omnipräsenten Endzeitstimmung nachzeichnet und die Welt ihrem Untergang entgegengeht, findet sich Thomas Glavinics Protagonist in Die Arbeit der Nacht (2006) ${ }^{10}$ bereits als letzter Mensch auf Erden vor.

Allerdings steht nicht nur die ständige Katastrophe im Fokus; auch oder gerade aufgrund dieser unablässig präsenten Desaster befindet sich die aktuelle Generation auf der Suche nach der eigenen, wie auch der gesellschaftlichen Identität. Die permanente Existenzangst wird zusätzlich genährt durch eine tief im Menschen verwurzelte Sinnsuche, die zudem Fragen nach dem eigenen Ich und dem eigenen Wert in der Welt aufwerfen. Während sich ältere Generationen noch dank vorhandener gesellschaftlicher Folien als zum Beispiel ,Nachkriegsgeneration“ oder ,1968er' definieren konnten, scheinen solche prägenden und identitätsbündelnden Bewegungen und Ereignisse mittlerweile zu fehlen - als ,Internet- und Mediengeneration" befindet sich die moderne Zivilisation angesichts einer kaum zu bewältigenden Informationsflut im stetigen Wandel. Zwischen Katastrophenüberfluss und Veränderungen verliert das Individuum an Halt und wird in der Anonymität als ,Humankapital in die Entindividualisierung getrieben.

Und das auch weil Katastrophen im heutigen (aufgeklärten) Verständnis allgemein nicht mehr, wie beispielsweise noch bezogen auf biblische Desaster (etwa die Sintflut), als ,Strafgericht Gottes“ verstanden

8. - Kathrin Röggla, „Geisterstädte, Geisterfilme“, in: Dies., a.a.O., S. 17.

9. - Sibylle Berg, Ende gut, Reinbek bei Hamburg, Rowohlt Taschenbuch Verlag, 2006.

10. - Thomas Glavinic, Die Arbeit der Nacht, München, dtv, 2013. 
werden. Seit dem Erdbeben von Lissabon 1755, unter anderem literarisch verarbeitet durch Heinrich von Kleist in der Erzählung Das Erdbeben in Chili (1806), ist deutlich, dass die sogenannte ,beste aller Welten' nicht zwischen gerechten und ungerechten Katastrophen unterscheidet, sowie das Leid zum Leben des Menschen stets dazu gehörtt11.

Es stellt sich nun die Frage, inwieweit sich jene globalen und auch lokalen Katastrophen, wie auch die zunehmende Haltlosigkeit in einer sich ständig verändernden Welt auf das Individuum niederschlagen und bei jenem schließlich in individuelle Krisen zwischen Zukunftsangst und Lust am Untergang der bestehenden Welt ausarten. Exemplarisch wird dies im Folgenden an den Berg und Glavinic aufgezeigt.

\section{„Die Unsicherheit, in der wir alle leben“ - Sibylle Bergs Ende gut}

Die Alltäglichkeit der Katastrophen zeigt sich besonders deutlich in Sibylle Bergs 2004 publiziertem Roman Ende gut, der eine namenlos bleibende Protagonistin zur „Heldin“ hat - sie verkörpert die durchschnittliche Deutsche in der Mitte ihres von Passivität und Trägheit geprägten Lebens. Ihre Namenlosigkeit unterstreicht dabei nicht nur die allgemeine Funktion als jede erdenkliche ,Person von nebenan', sondern auch zugleich ihre fehlende Individualität. Dem Jugendwahn und der Oberflächlichkeit zu Beginn des neuen Jahrtausends (durchaus in Anlehnung an die Popliteratur) weiß sie nichts entgegenzusetzen und so lässt sie sich, ohne bewusste Aktionen und in vollkommener Konformität, treiben:

Ich mache fast alles für Geld, und nichts davon interessiert mich. Ich habe mich für kaum etwas bewußt entschieden, ich habe es passieren lassen, das Leben. [...] Ich lebe alleine, weil das auch so passiert ist. [...] Ich mache die meisten Dinge, weil alle sie so machen ${ }^{12}$.

Den Anschluss an andere Menschen hat sie längst verloren, doch auch in ihrem eigentlich selbst gewünschten Alleinsein fühlt sie sich verloren und im Leben gescheitert. Der schier unendlichen Informationsflut, der sie sich medial allgegenwärtig ausgesetzt fühlt, versucht sie durch „Infohaufen“ Herr zu werden - doch auch diese Bemühung, die Welt um sich herum zu ordnen und Struktur zu verleihen, ist angesichts der unendlichen Masse zum Scheitern verurteilt. Die Resignation der

11. - François Walter spricht beispielsweise von einem ,,neue[n] Zeitalter in der Risikowahrnehmung“, das gravierende kulturelle Folgen nach sich zog und das Katastrophenbewusstsein für immer verändert hat. In: Ders.: Katastrophen. Eine Kulturgeschichte vom 16. bis ins 21. Jahrhundert, Stuttgart, Reclam, 2010, S. 23.

12. - Sibylle Berg, Ende gut, a.a.O., S. 15. 
Protagonistin schlägt sich in ihrer Negierung jeglicher Sinnhaftigkeit der eigenen Existenz nieder: „Wir sind der evolutionäre Sondermüll der Geschichte"13.

Dies zeigt sich vorranging in den Lebensweisen der Einzelpersonen, die in „O-Tönen“ zu Wort kommen und dem Leser ihre Resignation und Orientierungslosigkeit angesichts ihrer verlorenen Hoffnungen, Wünsche und Träume darlegen. „DIESE LEERE“14 scheint unauffüllbar, während angesichts der knappen Restlebenszeit des Einzelnen weder Ziel, noch Chance vorhanden sind. ${ }^{15}$ Als Teil der ,erste[n] Generation, die für ihr Überleben nicht zwingend arbeiten mußte" 16 , fehlt ihr das notwendige identitätsbildende Gesellschaftsereignis, das sie als Teil einer Gemeinschaft auszeichnen könnte - während dieser „Nichtzeit"17, in der sie lebt, ist der Untergang aller selbst im alltäglichen Leben fortdauernd spürbar. Die Anschläge auf das World Trade Center, ungefähr zwei Jahre vor Handlungsbeginn des Romans ${ }^{18}$, werden zwar von der Protagonistin mehrfach beiläufig als handlungsauslösendes Moment des weltweiten Untergangs ausgemacht ${ }^{19}$, doch schlussendlich identifiziert sie die Menschheit selbst als Wurzel allen Übels:

Alles bricht auseinander, das hat nicht mit den Twin Towers begonnen, sondern mit dem Beginn der Menschheit, es hat gegärt, gebrodelt, und nun lösen sich alte, nie sicher gewesene Werte auf, so daß keiner weiß, wie man eine Position beziehen soll ${ }^{20}$.

Der Untergang der Menschheit ist im täglichen Leben allgegenwärtig spürbar, auch wenn an der individuellen Alltagsroutine festgehalten und lethargisch das Weltende erwartet wird; durch die ständigen Anschläge befürchtet die Protagonistin allerdings den Übergang in eine „Phase des bedingungslosen Hasses, da jeder ein Opfer zu betrauern hat" 21 . Noch scheint dies weit entfernt: „In Zeitlupe findet eine Katastrophe nach der anderen statt, aber es beeinflußt nichts, ändert nichts. Wir machen weiter wie immer" 22 .

Zwei einschneidende Ereignisse im Leben der Protagonistin erschüttern diese in ihrem passiven Lebensentwurf allerdings so nachhaltig, dass sie beschließt, ihre Existenz in die Hand zu nehmen und grund-

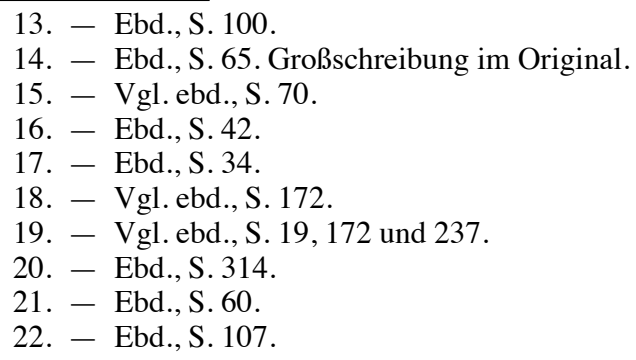


legend zu ändern: Nicht nur, dass sie selbst einen Terroranschlag erund vor allem körperlich unbeschadet überlebt, sie leistet zudem einer ihr völlig unbekannten Nachbarin, die durch ein solches Attentat ihre Kinder und damit jeglichen Lebensinn verloren hat, Sterbehilfe.

Bis zu diesem Zeitpunkt ist der Begriff der „Heldin“, wie er im gesamten Verlauf durch eine extradiegetische, unbestimmt bleibende Erzählinstanz in den Kapitelüberschriften für die Protagonistin gewählt wird, gegen den Strich besetzt - sie weist zu Beginn weder typische Eigenschaften einer solchen auf, noch vermag sie lange Zeit überhaupt für sich oder andere aktiv zu werden. Abgesehen davon, dass sie die Protagonistin und autodiegetische Erzählerin der Handlung ist, ist sie kaum ,jemand, der sich mit Unerschrockenheit und Mut einer schweren Aufgabe stellt, eine ungewöhnliche Tat vollbringt, die ihm Bewunderung einträgt" 23 . Die genannten Ereignisse fungieren jedoch als Wendepunkt: Erstmals stellt sie ihr bisheriges Leben in Frage, bedauert ihren Kontaktarmut und das verpasste Auskosten von Chancen. Sie erkennt den Wert ihres Lebens, was sie dazu veranlasst, endlich aktiv zu werden, ihr Heim zu verlassen und sich in die Welt hinaus zu begeben. Diese Initiationsreise, die einer Sinnsuche gleicht und an deren Ende sie zu einem neuen Selbst gelangt, führt sie durch verschiedene deutsche Städte bis in ihre Geburtsstadt Weimar, weiter in die Niederlande und schließlich nach Finnland. Die Protagonistin muss allerdings erst verschiedene Etappen und Erlebnisse meistern, bevor sie schlussendlich ihren Frieden mit sich und der Welt machen kann.

Die „Heldin“ durchquert nun eine Welt am Rande ihres Untergangs, die bevölkert ist mit einer unüberschaubaren Vielzahl an Menschen, die sich nicht als Teil einer Gemeinschaft fühlen; als Individuen sind sie stattdessen in dem Gedanken verfangen, einzigartig 24 und besonders zu sein : ,Sie denken noch immer, es sei Spaß, etwas, das sie nichts angeht die Krise, das Elend, die Anschläge, der Krieg -, wissen doch, daß ihnen ein besonderes Leben zusteht" 25 . Als sich die Ereignisse zuspitzen und Krieg, Terror und (tödliche) Krankheiten in der vermeintlichen sicheren westlichen europäischen Welt wüten, verharren die Menschen in einer „Mischung aus Panik und Verdrängung“ 26 - doch die Entladung der andauernd drohenden Katastrophe wirkt auf die Protagonistin geradezu erlösend, denn ,[d] as Gleichgewicht auf der Erde wird gerade wiederhergestellt - Elend für alle“27.

23. - http://www.duden.de/rechtschreibung/Held

24. - Vgl. Sibylle Berg, Ende gut, a.a.O., S. 206.

25. - Ebd., S. 94.

26. - Ebd., S. 146.

27. - Ebd., S. 172. 
Dieses tabula rasa ermöglicht zugleich einen Neuanfang, da alle Menschen am gleichen Ausgangspunkt stehen und die Chance erhalten, aus diesem gemeinsamen Katastrophenerlebnis heraus erst eine gesellschaftliche und daraus eine persönliche Identität zu entwickeln. Bereits Kathrin Röggla merkte an: ,Angeschlossen zu sein an die Hysterie da draußen ist immer noch besser, als ausgesetzt zu sein der Hysterie da drinnen, der Panik, die einen besetzt und deren Ursprung man nicht mehr orten kann“"28. Die Erfahrung der Katastrophe und das Bewusstsein des Überlebens ermöglicht überhaupt erst das Gemeinschaftserlebnis, das wiederum den Einzelnen als Konsequenz vor der Auseinandersetzung mit den eigenen Problemen schützt. Paradoxerweise braucht es also eine kollektive Erfahrung, über die sich das Individuum definieren kann und seine Identität als Einzelpersönlichkeit entwickelt.

Zusammenfassend lassen sich drei narrative Strategien erkennen, die Sibylle Bergs Roman Ende gut kennzeichnen: Durch die autodiegetische Instanz hat der Leser unmittelbar Anteil an den Gedanken, Gefühlen und Erlebnissen, die die individuelle Krise der Protagonistin auslösen und an denen, die sie jene überwinden lassen. Zum anderen erscheint der Text als Ganzes als Theaterinszenierung - so findet beispielsweise in der Mitte des Romans eine Pause statt, die durch eine nicht näher identifizierbare narrative Instanz kommentiert wird. Darauf folgt - ähnlich dem previously on... (précédemment dans...) einer Fernsehserie - eine Zusammenfassung der bisherigen Ereignisse, bevor es mit der eigentlichen Handlung weiter geht.

Darüber hinaus wird die autodiegetische Erzählung durch „O-Töne“ und „Informationshaufen“ ergänzt; in ersterem kommen chorartig andere Menschen (und teilweise personalisierte Elemente wie Kakerlaken, Hunde oder die Welt selbst) zu Wort. Auch jene sind ihrer Individualität beraubt, werden nur über ihre Eigenschaften wie „stiller Mann“ oder über Scheinnamen wie „Horst, der eigentlich Rüdiger heißt" definiert. Daraus wird erneut deutlich, dass identifizierende und das Individuum bezeichnende Namen angesichts der um sich greifenden Identitätslosigkeit an Bedeutung vollkommen verloren haben. Und auch die „Informationshaufen“, die Zeitungen, Flyern und ähnlichen Bekanntmachungen entnommen sind, tragen lediglich zur Veranschaulichung des katastrophal-chaotischen Zustands der Welt bei, dem nicht mehr durch Strukturierung und Ordnung Herr zu werden ist. Jene „Informationshaufen“ gestalten sich als postmoderne Collage von ,Fremdmaterial' und fangen sowohl die Stimmung einer Generation ein, wirken aber auch wie für ein Museum oder ein Archiv gesammelte Zeitdokumente und erzeugen somit Authentizität.

28. - Kathrin Röggla, „Die Rückkehr der Körperfresser“, in : Dies., a.a.O., S. 27. 
Zuletzt ist jedem Kapitel eine Überschrift vorangestellt, die allerdings über diese Funktion weit hinaus geht und sowohl zusammenfassend, als auch wertend das folgende Geschehen wiedergibt. Dies erinnert dabei nicht nur stark an Giovanni Boccaccios Decamerone (13491353), sondern auch an die lakonischen Kapitelüberschriften aus Hans Falladas Kleiner Mann - was nun? (1932).

Somit gelingt es Sibylle Bergs Roman, eine apokalyptische Untergangsstimmung einzufangen, die in ihrer Alltäglichkeit nahezu beiläufig erscheint. Die Protagonistin kann dem Ende der Welt nicht entfliehen, sondern sich nur an einen noch unberührten Ort (eine finnische Insel) zurückziehen, wo sie nach ihrer langen Reise endlich Menschen findet, die einen ähnlichen Lebensstil wie sie selbst pflegen. Das märchenhafte Ende symbolisiert zugleich die „Heldin“ selbst, die den Frieden mit sich gefunden hat und in einer für sie beruhigenden Zweisamkeit mit einem Mann leben kann. „Und sie leben weiter, bis an ihr Ende. Und zwar gut" ${ }^{\prime 29}$.

\section{„Weit und breit kein Mensch.“ - Thomas Glavinics Die Arbeit der Nacht}

Während die Isolation des Einzelnen in Ende gut noch selbstgewählt (und doch keine Lösung) war, ist sie in Thomas Glavinics 2006 erschienenem Roman Die Arbeit der Nacht von Anfang an erzwungen und ausweglos, was die Drastik für den Protagonisten Jonas nur verschärft. Das Desaster gestaltet sich ebenfalls auf vollkommen andere Weise: Jonas wacht eines Morgens auf und stellt nach einiger Zeit auf dem Weg zur Arbeit fest, dass alle Menschen und Tiere spurlos verschwunden sind: „Weit und breit kein Mensch. [...] Plötzlich fiel ihm auf, daß nicht einmal Vögel zu sehen waren"30. Wasser und Strom funktionieren auch weiterhin, allerdings ist das Internet zusammengebrochen und auch sonst empfängt kein anderes Kommunikationsmedium ein Signal geradezu fatal für die ,Generation Internet', die es gewohnt ist, jederzeit auf Mobiltelefone und das World Wide Web zugreifen zu können.

Allen Erklärungsversuchen zum Trotz stellt sich keine Lösung ein - die Unsicherheit und Vieldeutigkeit der (verschlafenen) Katastrophe spiegelt sich narratologisch: Es drängen sich verschiedene Lesarten auf, allerdings wird keine davon aufgelöst, wodurch der Deutungsspielraum bis zum Ende erhalten bleibt. Möglich wäre ebenso die Erklärung eines (Alb)Traums, festzumachen etwa am Motiv des Fallens, wie auch die eines (krankheitsbedingten) Wahnsinns, was sich an der zunehmend gehäuften Einnahme zahlreicher Tabletten ablesen lässt und auch mit

29. - Sibylle Berg, Ende gut, a.a.O., S. 335.

30. - Glavinic, Die Arbeit der Nacht, a.a.O., S. 11. 
der Schlaflosigkeit und Paranoia des Protagonisten zu erklären ist. In diesem Zusammenhang wäre ebenso ein Drogenrausch denkbar oder aber auch der Aufenthalt in einer Art (Vor)Hölle, in der sich der Protagonist allein nach seinem Tod befindet. Glavinics Roman steht dabei sicherlich in einer Tradition mit Marlene Haushofers Die Wand (1968) und Herbert Rosendorfers Großes Solo für Anton (1976), wobei dort beide Protagonisten durch tierische Begleiter nicht vollkommen allein und isoliert von anderen Lebewesen bleiben - denn, wie Jonas sich vergewissert, sind sogar alle Tiere des Zoos aus ihren Käfigen verschwunden ${ }^{31}$.

Im direkten Vergleich zu Bergs Ende gut begibt sich die Figur auch hier auf eine durch die äußeren Umstände angetriebene Reise, wobei es sich nicht um eine Initiationsreise, sondern vielmehr um eine Selbstvergewisserung, tatsächlich der letzte Mensch zu sein, und Suche nach anderen Menschen (im späteren Verlauf besonders nach Marie, seiner geliebten Lebenspartnerin) handelt. Während der Ausgangspunkt in Ende gut unklar bleibt, da lediglich von einer ,deutschen Stadt“32 gesprochen wird und durch die Reise der Protagonistin Hamburg, Berlin und Weimar, sowie weiter weg liegende Städte wie beispielsweise München oder Stuttgart ausgeschlossen werden können, beginnt und endet Die Arbeit der Nacht in der geschichtsträchtigen österreichischen Hauptstadt Wien.

Im Gegensatz zur namenlosen „Heldin“ bei Sibylle Berg verfügt aber Jonas durchaus über einen ihn identifizierenden (und sogar biblisch aufgeladenen) Namen. Allerdings stellt sich zugleich die Frage, was Identität überhaupt noch bedeutet, wenn man der letzte Mensch auf Erden ist und somit niemand da ist, von dem man sich abgrenzen oder mit dem man sich vergleichen kann - oder wenn schlichtweg niemand mehr da ist, der den Namen rufen kann. Und nicht zufällig erfährt man beispielsweise auch zu keiner Zeit den Namen der autodiegetisch erzählenden Protagonistin in Marlene Haushofers Die Wand.

Das Problem der Identität wird im Roman insofern auf die Spitze getrieben, als dass sich Jonas nach und nach in zwei Persönlichkeiten spaltet: Sobald er schläft, übernimmt ein fremdartig wirkender Teil seiner selbst die Oberhand über den gemeinsamen Körper und sabotiert Jonas' Pläne und Aktionen des Wachzustandes. Zu dieser Erkenntnis gelangt er erst spät - lange vergewissert er sich darüber, dass beispielsweise Türen abgeschlossen und Gegenstände wie von ihm abgelegt sind. Die erzwungene Isolation treibt ihn allerdings dazu, Videokameras zu platzieren und sich sowohl schlafend zu filmen, als auch Straßenzüge

31. - Ebd., S. 28f.

32. - Sibylle Berg, Ende gut, a.a.O., S. 15. 
und Plätze aufzunehmen. Geradezu gespenstisch wirkt das andere Ich, das Jonas nur als „den Schläfer“ bezeichnet:

Der Schläfer wälzte sich wie üblich von einer Seite zur anderen. Zuweilen erklang Schnarchen. [...] Der Schläfer warf die Decke ab. Jonas beugte sich vor. [...] Er verstand nicht, was er sah. Der Schläfer trug eine Kapuze. Zuvor hatte Jonas nicht genau hingesehen. Nun bemerkte er, daß der Kopf des Schläfers mit einer schwarzen Kapuze bedeckt war. Winzige Löcher für Augen, Nase und Mund waren hineingeschnitten. Der Schläfer setzte sich aufrecht auf die Bettkante. Die Arme an den Seiten aufs Bett gestemmt, saß er bewegungslos da. Er schien in die Kamera zu blicken. [...] Er saß da. Starr. Auf eine wortlose, ungeheure Weise lag Hohn und Herausforderung in seiner Haltung. Er $\mathrm{sa} ß$ herausfordernd da. [...] Jonas konnte nicht lange in diese Maske schauen. Er meinte in ein Loch zu blicken, seine Augen ertrugen die Leere nicht, er wandte sich ab. Sah wieder hin. Starre. Ein schwarzer Kopf. Lochgesicht. [...] Schwarzer Kopf, unbewegter Körper. Er saß da wie ein Toter. Langsam, wie in Zeitlupe hob der Schläfer den rechten Arm. Er streckte den Zeigefinger aus. Reckte ihn in Richtung Kamera. Verharrte ${ }^{33}$.

In der auktorialen Narration schlägt sich, wie an dieser Stelle besonders deutlich wird, nicht nur die Entfremdung Jonas' vom eigenen Ich, dem Unterbewusstsein, das im Schlaf in beispielsweise Träumen aktiv wird, wie auch vom eigenen Körper nieder, es spiegelt sich zugleich die für den Protagonisten geradezu ins Unerträgliche gesteigerte Anspannung wider - ebenso wie die Vermutung, dass Jonas schon längst tot ist. In Abgrenzung zu seiner wachen Identität, wird das schlafende Ich stets nur unpersonalisiert (und wieder wie bei Berg namenlos) versachlicht; „der Schläfer“" erscheint vielmehr als eine Art Geist, dessen Anblick für Jonas nicht lange auszuhalten ist. Bezeichnenderweise agiert Glavinics Schläfer ähnlich dem ,terroristischen Schläfer' im Verborgenen, um im geeigneten Moment zuzuschlagen - in diesem Fall im Untergrund der Psyche, dem Unterbewusstsein, womit sich der Feind im eigenen Inneren befindet. Auf diese Weise kann er als Sinnbild für die persönlichen (psychischen) Probleme des Protagonisten Jonas dienen. Ähnlich wie in Robert Louis Stevensons Erzählung Strange Case of Dr. Jekyll and Mr. Hyde (1886, dt. Der seltsame Fall des Dr. Jekyll und Mr. Hyde, frz. L'Étrange Cas du docteur Jekyll et de M. Hyde) nimmt auch er ein mysteriöses Medikament, hier mit Namen „Umirome“34.

33. - Glavinic, Die Arbeit der Nacht, a.a.O., S. 172-173.

34. - Der Autor hat zwei literarische Motive als Vorbilder genannt, die er in seinem Roman miteinander verwoben hat: Robinson Crusoe und Jekyll/Hyde. Vgl. in : Birgit Holzner: „Thomas Glavinics Endzeitroman Die Arbeit der Nacht", in : Evi Zemanek und Susanne Krones [Hrsg.], Literatur der Jahrtausendwende. Themen, Schreibverfahren 
Im Gegensatz zu Dr. Jekyll schluckt er dieses aber nicht, um sich $\mathrm{zu}$ verwandeln, sondern um die Verwandlung, also in diesem Fall schlichtweg seinen Schlaf, zu verhindern. Doch mit zunehmenden Schlafmangel und Medikamentenmissbrauch - er nimmt jegliche erdenkbare zu findende Tablette ein, weit über die empfohlene Dosis hinaus - steigert sich auch seine Paranoia, was wiederum dem Schläfer mehr Macht zukommen lässt. Die Persönlichkeitsspaltung kann somit nicht lange herausgezögert werden und kumuliert letztendlich im freiwilligen Suizid; so stürzt sich Jonas gemeinsam mit Maries Koffer und in Gedanken an sie vom Turm des Wiener Stephansdom - eine Schlussszene, die nicht zufällig an E.T.A. Hoffmanns Der Sandmann (1816) und dessen getriebenen Protagonisten Nathanael erinnert.

Die Verzweiflung schlägt wie bei Dr. Jekyll und Nathanael mehr und mehr in Panik und Wahnsinn um, so schreit er das Donnern eines Gewitters an ${ }^{35}$ oder vergewissert sich stets darüber, dass er wirklich allein war $^{36}$. In der Figur des Jonas zeigt sich allerdings vorrangig eines besonders deutlich - der Mensch ist nicht zur Isolation geschaffen. Als Gemeinschaftswesen brauchte er über Jahrtausende seiner Entwicklung hinweg stets die Anderen seiner Art zum Überleben; dieses Wissen hat sich tief in jeden Menschen eingebrannt. So kommt es beispielsweise, dass der Protagonist bewusst Geräusche erzeugt, obwohl er ,Lärm aller Art verabscheute“37, da er die quälende ,unnatürliche Stille“38 nicht mehr erträgt. Für ihn ist die beständige Ordnung seines Lebens aus dem Gleichgewicht geraten, die Geliebte ebenso verschwunden, wie der Vater, der ,nicht mehr da [war]. Wie alle anderen. Und wie alle anderen hatte er keine Spur hinterlassen“"39.

Das Problem ergibt sich für Jonas folglich in erster Linie daraus, dass die für ihn bisher als selbstverständlich erlebten bekannten und unbekannten Mitmenschen nicht nur verschwunden waren, sondern vielmehr dass dies ohne jeden Grund und Anzeichen geschehen ist. Der Protagonist stellt sich dabei in die Tradition des Topos des, verschlafenen Zeitenwandels', wie er in erster Linie durch Washington Irvings Rip van Winkle (1819) thematisiert wurde. Dies ist ein geradezu beliebtes Motiv im Rahmen ,Letzter Menschen'-Erzählungen und findet sich nicht nur in den bereits erwähnten Texten Die Wand und Großes Solo für Anton, sondern zum Beispiel auch in anderen postapokalyptischen Romanen wie George R. Stewarts Earth Abides (1949, dt. Leben ohne Ende, frz.

und Buchmarkt um 2000, Bielefeld, transcript Verlag, 2008, S. 221.

35. - Vgl. Glavinic, Die Arbeit der Nacht, a.a.O., S. 35.

36. - Vgl. ebd., S. 124.

37. - Ebd., S. 46.

38. - Ebd., S. 40.

39. - Ebd., S. 14. 
La Terre demeure) oder John Wyndhams The Day of the Triffids (1951, dt. Die Triffids, frz. Le Jour des Triffides).

Zugleich findet sich hier eine unverkennbare Anlehnung an Franz Kafkas Die Verwandlung (1912) ${ }^{40}$, in der sich der Protagonist Gregor Samsa ebenfalls nach seinem morgendlichen Erwachen mit für ihn vollkommen veränderten und mysteriös bleibenden Gegebenheiten arrangieren muss und die für ihn auch letztendlich den Tod bedeuten.

Geradezu überraschend schnell lässt Jonas jedoch zivilisatorische und kulturelle Konventionen hinter sich - ohne genauer zu wissen, was passiert ist, flaniert er vandalierend und plündernd durch Wien, beraubt Supermärkte und vor allem Apotheken und bricht in Wohnungen ein. Dies spricht für einen ausgebrochenen Egozentrismus, da er schon nach kürzester Zeit sein Überleben, oder vielmehr seine Annehmlichkeiten, priorisiert. Auch an dieser Stelle findet sich eine Parallele zu Sibylle Bergs Roman Ende gut: Genau wie die Menschen in ihrem Text, hat sich Jonas regelrecht herbeigesehnt, etwas Besonderes zu sein. Dies zeigt sich in seinem Fall an einer ausgeprägten Katastrophenlust -

Er hatte sich gewünscht, vor aller Augen durch eine Gefahr gegangen zu sein. Die Auszeichnung zu tragen, eine harte Prüfung bestanden zu haben. Er hatte ein Überlebender sein wollen. Ein Auserwählter hatte er sein wollen. Der war er jetzt ${ }^{41}$.

Die Krise des Individuums Jonas zeigt sich in seiner zunehmenden Identitätsstörung. Im Gegensatz zu Sibylle Bergs Protagonistin geht er den umgekehrten Weg und verliert seine eigene Identität, die sich in sein Wach- und sein „Schläfer"-Ich aufteilt, zunehmend. Er stellt dazu fest:

Ein Leben. Man war nur ein oder zwei oder drei Jahre derselbe, dann hatte man mit der früheren Persönlichkeit, mit der vor vier Jahren, immer weniger gemein. [...] Er hatte einmal Briefe gefunden, die er zehn Jahre zuvor einer Freundin geschrieben, aber nie abgeschickt hatte. Der da schrieb, war ein ganz anderer. Eine andere Persönlichkeit. Nicht ein anderes Ich. Denn das blieb zu allen Zeiten gleich ${ }^{42}$.

Dies bedeutet dann allerdings im Umkehrschluss, dass die „Schläfer"-Seite in ihm schon Zeit seines Lebens angelegt war und ein untrennbarer Teil von ihm darstellt. Geht man von der These aus, dass eben jene nächtliche Persönlichkeit - gleich der Jekyll'schen Abspaltung - seine unterbewussten Probleme verkörpert, so bringen ihn jene Probleme, denen er sich angesichts seiner völligen Isolation ausgesetzt

40. - Vgl. in: Wolfgang Müller-Funk: „Nach der Postmoderne: Thomas Glavinic“ apokalyptischer Roman Die Arbeit der Nacht", in: GegenwartsLiteratur, 2010, 9, S. 14.

41. - Glavinic, Die Arbeit der Nacht, a.a.O., S. 94.

42. - Ebd., S. 392. 
sieht, im wahrsten Sinne des Wortes zu Fall. Jonas ist nicht mehr Teil einer Gemeinschaft und wie bereits dargelegt, erklärt Kathrin Röggla ja gerade die Katastrophenlust des Einzelnen aus dem Angeschlossensein an ein größeres Ganzes ${ }^{43}$. Dies trifft genau auf den Protagonisten in Die Arbeit der Nacht zu, der nun nicht mehr auf die alltäglichen Katastrophen ,da draußen“44 zugreifen kann und sich daher gezwungen sieht, sich mit seiner „Hysterie da drinnen“ 45 auseinander zu setzen.

\section{Fazit}

„Für einige Autoren ist die Lust an Katastrophen ein konstitutiver Bestandteil der Kultur der Postmoderne“ 46 - François Walter fasst in seiner Kulturgeschichte der Katastrophe zusammen, inwiefern sich die momentane Gesellschaft so sehr für Desaster faszinieren lässt. Die Verarbeitung einschneidender Ereignisse fand von jeher auch - oder gerade vorrangig - auf dem literarischen Feld statt. Und gerade die Postmoderne hat, so scheint es, das Lebensgefühl ,Katastrophe verinnerlicht - was vor allem von Kathrin Röggla postuliert wird: Die mediale Überreizung und die Banalisierung schlimmster Ereignisse führt (zwangsläufig) zu Abstumpfungserscheinungen und Alltagserleben. So ist es nicht verwunderlich, dass sich gerade angesichts krisenhafter Zeiten und den weltverändernden Anschlägen des 11. September 2001 eine jüngere Schriftstellergeneration mit der Gesellschaft und der Kultur ihrer Zeit auseinandersetzt und jene Beobachtungen wie auch Prognosen Eingang in das Schaffen finden.

Zugleich bleibt die Lust an den Katastrophen erhalten; durch die mediale Überreizung wurde jeder Mensch bereits Zeuge von eingestürzten Fabriken in Bangladesh bis hin zu Tsunamiopfern in Japan oder erschossenen Flüchtlingen in Syrien. Dies bedeutet zugleich eine gewisse Distanz, da diese Menschen dem Zuschauer fremd bleiben und er sich in seinem (westlichen) Zuhause in Sicherheit wiegen kann - das Prinzip des (fast voyeuristischen) Probehandelns funktioniert für ihn auch weiterhin.

$\mathrm{Zu}$ diesen postmodernen literarischen Katastrophentexten zählen auch Sibylle Bergs Ende gut und Thomas Glavinics Die Arbeit der Nacht. Beide Romane greifen dezidiert Einzelschicksale heraus, deren individuelle Krisen der Leser aus nächster Nähe verfolgen kann und deren Lösungsmöglichkeiten er begleitet. Bergs Protagonistin erkennt,

43. - Vgl. Kathrin Röggla, „Die Rückkehr der Körperfresser“, in: Dies., a.a.O., S. 27.

44. - Ebd.

45. - Ebd.

46. - François Walter: Katastrophen. Eine Kulturgeschichte vom 16. bis ins 21. Jahrhundert, Stuttgart, Reclam, 2010, S. 20. 
dass der ständige Drang der Menschen nach Selbstbestätigung, das Zentrum der Welt zu sein zu wollen ${ }^{47}$, aus der ständigen Suche nach dem Sinn der eigenen Existenz und dem Wunsch, nach Bedeutung resultiert. Dies spiegelt sich auch in der Figur des Jonas wider, der sich wiederholt wünschte, ein Überlebender beziehungsweise Auserwählter zu sein und der sich schließlich unfreiwillig in einer solchen Situation wiederfindet ${ }^{48}$. An ihm zeigt sich das zunehmende Zerbrechen des Individuums, das als Gruppentier ohne menschlichen oder gar tierischen Kontakt nicht lebensfähig ist und dessen Alleinsein mit dem eigenen Ich tödlich endet.

Doch während Jonas an der neuen Situation scheitert, gelangt Bergs namenlose Protagonistin zu einem , guten Ende', in dem sie sich zwar weitestgehend von der untergehenden Welt zurückzieht und trotzdem Teil einer kleinen Inselgemeinschaft ruhiger Menschen bleibt. Das ,Ende' ist bei beiden Texten relativ - es bedeutet stets nur das Ende einer, nicht der Zivilisation. Während der Leser Jonas noch vor seinem endgültigen Tod verlässt, ist auch der allumfassende Untergang in Ende gut nicht eingetreten. Die Katastrophenzeit ist vor allem im neuen Jahrtausend allgegenwärtig und daher auch selbst wieder relativierbar - handelt es sich tatsächlich noch um eine Katastrophe, wenn sie Innen (im Individuum) und Außen (in der Welt) schon zum Normalzustand geworden ist?

Das Individuum scheint allerdings angesichts der omnipräsenten Endzeitatmosphäre, die zum Dauerzustand verkommen ist, einer Krisenstimmung verfallen zu sein - sei es aufgrund des Zusammenbruchs der bisher bekannten und daher auch so bequemen Gesellschaftsstruktur, oder aber aufgrund der Frage nach dem eigenen Selbst in der Auseinandersetzung mit einem möglichen abgespaltenen Ich.

Losgelöst von der Gesellschaft ist keiner der beiden Protagonisten in der Lage zu (über)leben, und dennoch bleibt die Frage nach dem Wert der eigenen Identität bestehen; ist sie in jenen unsicheren Zeiten belanglos und ignorierbar (so bleiben Bergs Figuren überwiegend namenlos oder auf austauschbare Eigenschaften beschränkt) oder aufgrund einer Überindividualisierung (wenn es nur noch das eigene Ich gibt, auf das alles zurückgeworfen wird) unerträglich? So wundert sich auch Jonas, dass der plötzliche Tod Tausender nicht von allen anderen Menschen wahrgenommen wird und schließt daraus: „Das Prinzip zählte, nicht der einzelne. Entweder sie waren alle verurteilt. Oder keiner"49. Ende gut?

47. - Vgl. Sibylle Berg, Ende gut, a.a.O., S. 269.

48. - Vgl. Glavinic, Die Arbeit der Nacht, a.a.O., S. 94.

49. - Ebd., S. 390. 